\title{
Civilisations
}

Revue internationale d'anthropologie et de sciences

humaines

43-2 | 1996

Problèmes africains contemporains

\section{Le retrait des États-Unis d'Amérique de l'UNESCO (1984)}

Conséquences et plaidoyer pour le retour à l'universalité

\section{René-Pierre Anouma}

\section{(2) OpenEdition}

Journals

Édition électronique

URL : http://journals.openedition.org/civilisations/1572

DOI : $10.4000 /$ civilisations. 1572

ISSN : 2032-0442

Éditeur

Institut de sociologie de l'Université Libre de Bruxelles

\section{Édition imprimée}

Date de publication : 1 avril 1996

Pagination : 111-160

ISBN : 2-87263-160-7

ISSN : 0009-8140

Référence électronique

René-Pierre Anouma, «Le retrait des États-Unis d'Amérique de l'UNESCO (1984) », Civilisations [En ligne], 43-2 | 1996, mis en ligne le 29 juin 2009, consulté le 20 avril 2019. URL : http://

journals.openedition.org/civilisations/1572; DOI : 10.4000/civilisations.1572 


\title{
Le retrait des Etats-Unis d'Amérique de I'UNESCO (1984) : \\ conséquences et plaidoyer pour le retour à I'universalité
}

\author{
René-Pierre ANOUMA \\ Université d'Abidjan
}

\section{Summary}

Taking part in the solutions of main problems at the dawn of the third millennial, peace, development and environnemental protection in the world appears like an imperative of the international community. In so far as instrument of intellectual cooperation, Unesco plays an essential role.

The recent debates about its role and missions involved the $1984-85$ crisis that led to the withdrawal of three members states and particularly two founder members : the United States of America and the United Kingdom.

This article deals with the withdrawal processus of the United States of America, the main contributor of the Organization and analyses the efforts made by both directors general the former one Mr. Amadou Mathar M'Bow as well as the present one Mr. Federico Mayor to preserve Unesco universality.

\section{Introduction}

L'histoire de la création de l'Organisation des Nations-unies pour l'éducation, la science et la culture fait apparaître le rôle fondamental joué par les occidentaux et plus 
particulièrement par le Royaume Uni, la France et les États-Unis d'Amérique.

Convoquée par les gouvernements du Royaume Uni et de la France, la conférence préparatoire qui se tint à Londres du ler au 16 Novembre 1945 regroupait quarante quatre pays réunis "sur un pied d'égalité, avec une voix pour chacun"1. Le principe de l'égalité des États était ainsi clairement affirmé. Pour René Cassin qui présidait la délégation française la future organisation se devait "de créer un climat international de confiance et de paix" mais aussi "de créer l'esprit de paix dans le monde"2

Plusieurs initiatives anglo-saxonnes et françaises, à l'origine de la conférence préparatoire, doivent être rappelées. Celles de Richard Butler, président du Board of Education du Royaume Uni et de Sir Malcolm Robertson, président du British Council qui, en 1942, invitèrent les ministres de l'Éducation des pays alliés à se réunir à Londres en vue de réfléchir et d'aider à la reconstruction de leurs systèmes éducatifs détruits par la guerre; celle du Français René Cassin ensuite qui constitua un comité chargé d'examiner trois propositions dont deux réalisées par les États Unis et le Royaume Uni visant à créer un nouvel organisme international ayant en charge les questions éducatives et culturelles.

C'est aux anglo-saxons que nous devons la célèbre phrase du premier alinéa du préambule de l'Acte constitutif : "Les guerres prenant naissance dans l'esprit des hommes, c'est dans l'esprit des hommes que doivent être élevées les défenses de la paix". En effet avancée par le Premier ministre britannique Clément Attlee, elle fut énoncée par l'américain Archibald Macleish, qui dirigeait la délégation des États Unis, elle fut partagée par l'ensemble de la conférence. C'est à une Britannique également, Mademoiselle Ellen Wilkinson, ministre de

I l'UNESCO à la veille de son quarantième anniversaire, ouvrage réalisé sous la direction de A.M.M'BOW, UNESCO, 1985, p.13.

2 ibidem, op. cit, p13 
l'Éducation de l'Angleterre et du Pays de Galles, chef de la délégation du Royaume Uni et président de la conférence que nous devons la place de la science dans l'intitulé de l'Organisation. Son premier directeur général Julian Huxley était aussi ressortissant du Royaume Uni.

C'est à ce dernier que l'Unesco doit son premier programme qu'il voulait voir repoer sur "une philosophie exempte de tout sectarisme... globale et universelle, fondée sur un humanisme mondial, scientifique, évolutionniste".3. Pour Julian Huxley en effet "l'Unesco a besoin de fonder son action sur une philosophie, une hypothèse de travail qui tende à expliquer les buts et les fins de l'existence humaine et qui puisse dicter, ou tout au moins suggérer une prise de position devant les différents problèmes. Si elle ne possède pas une conception philosophique de ce genre, qui lui permette d'envisager les choses sous un angle unique, l'Unesco, avertissait-il, risquera de prendre des mesures fragmentaires, ou même contradictoires. Il lui manquera en tout cas le principe directeur de l'élément d'inspiration que fournit la croyance en une doctrine générale cohérente 4 .

Monsieur Amadou Mathar M'Bow près de quarante ans plus tard ne dit rien d'autre lorsqu'il observe dans la présentation qu'il fait du projet de Plan à moyen terme en 1982 que "face à la complexité mouvante du monde actuel, à l'interdépendance des problèmes, à l'enchevêtrement des tendances, à la multiplicité des modes de lecture des événements, il n'est guère possible de penser correctement les problèmes de l'éducation, de la science, de la culture, de la communication et de l'information sans des références solides au contexte général dans lequel ils se situent"s. Il en est de même lorsqu'il parle du besoin ressenti par la communauté internationale d'une étude continue des grands

\footnotetext{
3 ibidem, op. cit p. 17

4 ibidem, op. cit p. 25

5 La problématique mondiale et les orientations du 2e Plan à moyen terme (19841989), Paris, avril 1982, pp. 21 à 59.
} 


\section{Problèmes africains contemporains}

problèmes menée à partir d'un lieu, l'Unesco, où s'équilibreraient les tendances diverses qui se manifestent au sein de cette communauté et qui serait par vocation celui de la connaissance intellectuelle et non plus celui de telle ou telle préoccupation spécifique ${ }^{6}$.

Julian Huxley ne fut pas suivi dans son interprétation des buts et des fonctions de l'Unesco, mais la commission préparatoire dont il était le secrétaire exécutif décidera que son étude serait publiée comme document séparé, signé et considéré comme exprimant le point de vue personnel de son auteur. Les idées de Julian Huxley n'en cessèrent pas moins d'alimenter les débats et les oppositions entre les différents courants de pensée. L'Unesco de son côté n'en continuait pas moins de servir la coopération internationale puisque ses activités ont prouvé qu'il n'existait aucun conflit entre sa vocation éthique et le concours pratique et concret qu'elle pouvait apporter aux États membres. N'était-ce pas le voeu qu'exprimait Jacques Marltan, chef de la délégation française à la Conférence générale de Mexico en 1947 lorsqu'il déclarait que "...le dernier réduit de l'accord des esprits (était) assez pour entreprendre une grande oeuvre" ?

En écartant l'idée d'une philosophie qui serait celle de l'Organisation et fonderait son action et en se ralliant, à titre de compromis, à la notion de "commune pensée pratique" avancée par le Français, les pères fondateurs ne signifiaient-ils pas ainsi la volonté de leurs gouvernements respectifs de préserver leurs options nationales - politiques, idéologiques et culturelles ? N'était-ce pas affirmer le caractère politique de l'Organisation que confirmera, au reste, la réforme en 1954 du Conseil exécutif, à l'initiative du gouvernement des États-Unis il faut le rappeler ; réforme au terme de laquelle les membres du Conseil exécutif représenteraient leur gouvernement?

Partisans de l'idéologie et tenants d'une conception plus pragmatique de l'Organisation s'opposent encore aujourd'hui. La

6 ibidem op cit.25 
grave crise que vient de vivre l'Unesco n'est que l'expression de ces divergences fortement amplifiées par le retrait de deux États membres fondateurs : le Royaume Uni et les États-Unis d'Amérique. Ainsi ces deux pays qui ont joué, au lendemain de la Seconde Guerre Mondiale, un rôle de premier plan dans la création des institutions et structures multilatérales, dont l'Unesco, prenaient le risque de menacer ces dernières ! Mais comment auraient-ils pu concéder à Monsieur M'Bow, fut-il l'interprète d'une majorité d'États membres mais qui dans la réalité fournissent à peine $6 \%$ des ressources financières de l'organisation, ce qu'ils avaient refusé au britannique Julian Huxley ? Pouvaient-ils par ailleurs céder aux pressions, telles celles exercées par des mouvements comme "les Américains pour l'universalité de l'Unesco" ou "les Amis de l'Unesco" au Royaume Uni ? Quelle chance de pouvoir infléchir leur décision avaient les actions entreprises au sein du "comité pour l'universalité de l'Unesco par des intellectuels américains comme Linus Pauling, des personnalités comme Robert Mc Namara ou J.K. Galbraith, voire de nombreux lauréats de Prix Nobel ?

Ce n'était pas la première fois que des États membres décidaient de quitter l'Organisation ou que des directeurs généraux soient contraints à la démission. Nous avons celle du mexicain Jaimes Torres Bodet, directeur général qui se retira en 1950 faute d'avoir pu obtenir l'accroissement du budget qu'il souhaitait pour conduire efficacement le programme de l'Organisation.

Quant aux retraits des États membres, l'Unesco avait enregistré ceux de la Pologne et de la Hongrie en Décembre 1952, de la Tchécoslovaquie en Janvier 1953, de l'Union sud-africaine en 1955 et du Portugal en 1971. En Janvier 1965, l'Indonésie déposait un préavis de retrait qui fut annulé un mois plus tard. Tous ces États à l'exception de l'Union sud-africaine ont regagné par la suite l'Organisation. Trois de ces États, la Pologne, la Tchécoslovaquie et l'Union sud-africaine étaient représentés à la réunion des ministres de l'Éducation à Londres en 1942 et à la conférence préparatoire de Novembre 1945 à laquelle ne 
participait qu'un seul Etat africain au sud du Sahara alors indépendant, le Libéria.

La décolonisation et l'accession des peuples colonisés à l'indépendance et à la vie internationale se traduiront par une évolution quantitative des États membres. L'Organisation s'enrichira de leur spécificité culturelle mais en même temps devra faire face, dans ses domaines de compétence, à leurs préoccupations économiques et sociales, en un mot participer à leur développement.

Les activités de l'Unesco dans ce domaine vont s'inscrire dans le cadre de la résolution $1515(\mathrm{XV})$ de l'Assemblée générale des Nations unies adoptée en 1960. Cette résolution recommande en effet "...que l'assistance consacrée à la formation technique, à l'éducation et au pré-investissement, qu'elle émane d'organisations internationales ou de gouvernements pris individuellement, soit considérée comme un élément important du développement économique des pays sous-développés"7. L'éducation reconnue comme un investissement rentable et un facteur de développement économique propre à assurer l'épanouissement politique, économique, social et culturel du tiers monde, sera l'objet de soins attentifs puisque d'importantes ressources financières, matérielles et humaines lui seront consacrées.

Ainsi la coopération de l'Unesco avec les États membres en développement sera donc essentiellement axée sur l'éducation avec la création de diverses institutions de formation de maîtres, d'enseignants du secondaire, de gestionnaires ainsi que le renforcement des structures destinées à la production de matériels éducatifs adaptés aux caractéristiques nationales. Des unités chargées de la planification et de l'administration de l'éducation verront le jour, tandis que des cadres de concertation, d'échange d'idées, d'information et d'expérience, voire de définition de

${ }^{7}$ L'UNESCO à la veille de son quarantième anniversaire, op. cit p.25 
stratégies communes seront crées à l'initiative ou sous l'égide de l'Unesco.

En ce qui concerne l'Afrique, la première conférence d'États sur le développement de l'éducation qui s'est tenue à Addis-Abeba en 1961, la conférence sur l'avenir de l'enseignement supérieur en Afrique organisée à Tananarive en 1962, la conférence des ministres de l'Éducation et des ministres chargés de la Planification en 1964 à Abidjan ont été organisées sous les auspices de l'Unesco. L'Afrique lui doit également l'organisation de plusieurs conférences ministérielles régionales et sous-régionales qui ont abouti à la création et au renforcement de liens de coopération, liens qui se sont par ailleurs instaurés avec les organismes intergouvernementaux opérant dans le cadre de ses domaines de compétence : l'Organisation de l'Unité Africaine (OUA), mais aussi la Communauté Économique des États de l'Afrique de l'Ouest, le Comité permanent inter-États de lutte contre la sécheresse, l'Organisation africaine de la propriété intellectuelle, la Communauté économique des pays des grands lacs et l'Union panafricaine des télécommunications, la Commission Économique pour l'Afrique...

La fusion en 1966 du Programme élargi d'assistance technique et du Fonds spécial en un Programme des Nations unies pour le développement (PNUD) ne fera qu'accentuer cette tendance dans laquelle l'Unesco jouera de plus en plus un rôle de point focal.

C'est dire l'importance que revêt l'Unesco pour les pays en développement, ce qui expliquerait leur attachement pour la coopération multilatérale que symbolisent les Institutions spécialisées du système des Nations unies en général et plus particulièrement en ce qui nous concerne l'Organisation de la Place de Fontenoy.

En effet la grande transformation qu'a subie le système des Nations-unies est qu'il comporte aujourd'hui, avec une composition plus diversifiée, trois fois plus d'États membres qu'a 
l'époque de sa création. Cette nouvelle recomposition du contexte international a tout naturellement entraîné des situations d'une nature différente aussi bien au niveau des problèmes examinés que celui de ses différentes instances, de leur méthode de travail et des mentalités. Ainsi les majorités dont pouvaient se prévaloir les États membres fondateurs, les "grands" d'une façon générale, ne sont plus aussi évidentes. Jusqu'a la fin des années 50, c'était monnaie courante de se plaindre du veto de l'Union Soviétique au Conseil de sécurité. Ces dernières années ce sont les États Unis et parfois le Royaume Uni qui ont exerce le droit de veto. Or ce droit apparaît aujourd'hui comme anachronique, en tout cas contraire au principe d'égalité des États. A ce point de vue les Institutions spécialisées, à l'inverse de l'ONU, offrent le cadre d'une expression plus volontariste dans la mesure ou, avec l'inexistence d'un droit de veto et de majorité spéciale, chaque pays y dispose d'une voix de même valeur que les autres. Une thèse pouvant l'emporter dès lors qu'elle serait rationnellement présentée, c'est-à-dire de façon cohérente, diligente et persuasive.

Ainsi l'accusation de "politisation" régulièrement faite aux Institutions ne résulterait-elle pas du refus de la réalité, voire d'une incapacité des "grands" à mobiliser assez de voix pour empêcher ces Institutions de débattre de certaines questions ? Cette impuissance ne serait-elle pas à l'origine des critiques faites à l'encontre de la coopération multilatérale qui permet ce genre de débats et la faveur pour la coopération bilatérale sujette aux pressions, donc aux diktats?

La "politisation" de l'Unesco n'est pas récente et elle serait même le fait de ceux qui s'en plaignent. C'est en effet à l'initiative du gouvernement des États Unis en 1954 que les membres du Conseil exécutif qui exerçaient auparavant leurs pouvoirs au nom de la Conférence générale tout entière allaient dorénavant représenter leur gouvernement. Ils perdaient par la même occasion leur liberté d'opinion et d'action par rapport à ce dernier. Mais pouvait-il en être autrement dans une Organisation intergouvernementale ou les problèmes traités, l'éducation, la culture, la science et la communication, touchent directement aux 
intérêts des gouvernements et des États et à leurs diverses idéologies ? L'élaboration et l'adoption des différents Plans à moyen terme et notamment le deuxième qui nous intéresse ici, donneront l'occasion d'en vérifier la portée.

L'élaboration du deuxième Plan à moyen terme n'est pas étrangère à la crise de l'Institution. Elle l'accentuera même car des débats qu'elle a suscités émergeront deux conceptions du rôle et de la mission de l'Unesco. Cette évolution que d'aucuns considèrent comme situant l'Unesco en dehors des objectifs assignés par les pères fondateurs servit de prétexte aux retraits de trois États membres : les États-Unis d'Amérique, le premier contributeur avec $25 \%$ du budget, le Royaume Uni, le dépositaire de la Charte de l'Unesco et Singapour ; quand certains États membres, comme le Japon, n'en brandissaient que le spectre. Elle fut incontestablement à l'origine du départ de Monsieur Amadou Mathar M'Bow en 1987 après treize années passées à la tête de l'Institution.

En effet l'unanimité qui avait présidé en 1980 à sa réélection n'avait pas longtemps résisté à la controverse née de l'instauration du Nouvel ordre mondial de l'information et de la communication (NOMIC). Inspirée du rapport Mc Bride cette question avait été confiée à l'Unesco par l'Assemblée générale des Nations-unies. Bien que passée de mode l'Organisation en couvait encore les séquelles tant avaient été vives et passionnées les controverses qu'elle avait suscitées. Alors que Monsieur M'Bow présente le Nomic comme de nature à contribuer à instaurer une situation plus équitable dans le domaine de la communication et des médias, et ce grâce à des programmes de développement des infrastructures et de formation pour atténuer progressivement les inégalités existantes, l'homme va se retrouver au centre d'une violente polémique aussi bien sur sa personne, ses méthodes de travail, voire ses convictions religieuses que sur l'orientation "politique" qu'il aurait imprimée à l'Organisation. 
Le 2e Plan à moyen terme n'a été que prétexte à la crise puisque l'opposition entre les différentes visions du rôle et des fonctions de l'Unesco remonte aux origines de la création de l'Organisation.

Régulièrement évoquée, cette opposition va tout simplement connaître dans les années 1980 une tension particulièrement vive au point de provoquer le retrait des ÉtatsUnis d'Amérique en 1984. Nous examinerons les raisons avancées pour justifier ce départ dont les conséquences firent l'objet d'une session extraordinaire du Conseil exécutif en 1985.

\section{L'amplificateur de la crise: le 2 e plan a moyen terme}

Les débats que suscitèrent l'élaboration et l'adoption du $2 \mathrm{e}$ Plan à moyen terme avaient fait apparaître trois grandes tendances épousant en gros les traditionnels clivages Est-Ouest-Tiers monde.

Les pays occidentaux tout en approuvant dans son ensemble le Plan ont cependant estimé que certains de ses programmes, ceux notamment consacrés à la réflexion sur les problèmes mondiaux et les études prospectives, étaient inutiles, onéreux et surtout en dehors des traditionnels domaines de compétence de l'Unesco. Ils ont remis en cause la notion de "droit des peuples" au motif que l'Unesco, en débattant de cette question, voulait subordonner les droits de l'homme aux droits des peuples. Pour Monsieur M'Bow, au contraire, rien dans le programme proposé n'autorisait une telle interprétation. Pour lui en effet, et c'était bien le sens du programme, "droits de l'homme et droits des peuples sont organiquement liés. Sans les droits des peuples, les droits de l'homme risquent de rester purement 
formels... Sans droits de l'homme, les droits des peuples ne peuvent être un recours contre l'injustice ou la tyrannie"8.

Les pays socialistes ont, de leur côté, privilégié le problème de la paix, de la sécurité dans le monde et la compréhension internationale, quand les pays en développement, sans négliger ces questions, se sont principalement intéressés aux programmes liés à leur développement.

En effet, parce que la paix conditionne et garantit leur épanouissement, les pays en développement ont non seulement adhéré à ces thèmes, mais souhaité également que le Plan comporte un grand programme relatif aux pays les moins favorisés, et ce conformément au nouveau programme substantiel d'action pour les années 1980 en faveur des pays les moins avancés. Rappelons que ce programme avait été adopté par la Conférence des Nations-Unies sur les pays les moins avancés tenue à Paris du 1er au 14 Septembre 1981. Le Palais de l'Unesco en avait alors abrité les travaux.

Mais au-delà de ces tendances, reflet de la diversité et des préoccupations des États membres, ce sont en fait deux conceptions du rôle et de la mission de l'Unesco qui s'opposent.

\section{1 rôle et mission de l'UNESCO}

Les pays occidentaux et particulièrement les États-Unis estiment que l'Unesco devrait uniquement se cantonner dans son domaine de compétence. Il s'agit pour eux de l'alphabétisation dans le monde, le développement scientifique et la protection du patrimoine culturel mondial. Au lieu de cela, l'Unesco, estimentils, se réfugie dans l'abstrait et, péché suprême, fait de la

8 Document 115 Ex/INF.4 (prov). Présentation par le Directeur général du projet de Plan à moyen terme pour 1984-1989, p. 8. Voir également le document 115 EX/F.6 (prov). Réponse du Directeur général au débat relatif au projet de Plan à moyen terme pour 1984-1989, pp. 49-57. 
politique, puisqu'elle ambitionne de traiter de questions relatives à la paix dans le monde, au désarmement et à la sécurité. Toutes choses qui, selon eux, ne relèvent que de la compétence de l'Organisation des Nations unies.

La majorité des États, et ceci est apparu lors des débats, estime au contraire que la mission traditionnelle de l'Unesco n'exclut pas une réflexion préalable sur sa finalité et sur les grands problèmes mondiaux. Cette réflexion pouvant d'ailleurs contribuer au progrès des idées et à la prise de conscience, la signification et la portée de ces questions.

En fait cette réflexion était mise en cause par les occidentaux dans la mesure où elle mettait l'accent, voire légitimait des concepts et des notions comme l'identité culturelle, l'égalité des cultures, les droits des peuples et non plus seulement de l'homme, la dimension culturelle du développement... Elle débouchait, ce faisant, sur la remise en cause de tout un système de valeurs et de références, de concepts et de symboles, d'idées reçues et tenues jusque là pour immuables. Il n'en fallait pas plus pour que l'Organisation où était suscité un tel débat fut accusée de porter atteinte aux valeurs et aux institutions démocratiques occidentales, en un mot, à la suprême référence: la culture occidentale.

Mais à travers l'Organisation, c'est son directeur général, Monsieur Amadou-Mathar M'Bow qui se trouve visé en raison de la ligne imprimée à l'Unesco depuis son élection en 1974 ; ligne dans laquelle le gouvernement des États-Unis, en particulier, croit déceler un "antiaméricanisme viscéral" d'autant plus intolérable que ce pays participait pour $25 \%$ du budget de l'Unesco. La question du budget deviendra, au reste, un enjeu majeur de la crise.

\section{2. le budget : un moyen de pression}

Examinant le projet de programme et budget pour l'exercice 1984-1985, la première tranche d'exécution du Plan à 
moyen terme 1984-1989, le Conseil exécutif lors de sa session d'automne 1983 s'était prononcé en faveur d'une hypothèse de croissance positive, de l'ordre de 4 à $6 \%$, par rapport à la base de 1982-1983. Alors que la majorité des États membres se prononça en faveur de la proposition du directeur général, c'est-à-dire pour une croissance positive du budget, certains États et notamment les États-Unis défendirent la thèse d'une croissance zéro en termes réels.

Deux arguments essentiels fondaient leur raisonnement : la situation économique difficile qui imposait des politiques budgétaires rigoureuses et de sérieuses restrictions des dépenses publiques d'une part et d'autre part la nécessité d'un éventuel alignement du budget de l'Unesco sur celui des autres Institutions du système des Nations-unies.

Le Conseil exécutif, accédant aux propositions du directeur général, ne devait pas retenir ces deux arguments.

En ce qui concerne le premier, celui avancé pour exclure une croissance positive du budget, le directeur général s'est référé à certaines dispositions budgétaires et notamment le remboursement des sommes figurant au titre VIII du budget, en d'autres termes la réserve pour fluctuations monétaires. En se fondant sur l'exercice 1981-1983 le directeur général a fait observer qu'en réalité le montant de la contribution de chaque Etat membre exprimée en dollars courants devrait, au contraire, diminuer de 10,8\% en 1984-1985. C'était en particulier le cas pour les États-Unis. Aussi l'Etat qui avait versé la totalité de ses contributions en 1981-1983 n'aurait plus à payer en 1984 qu'une somme diminuée d'un montant correspondant à environ $20 \%$ de la contribution totale de l'exercice 1984-1985. Cette dernière étant elle-même inférieure de $10,8 \%$ en dollars courants à la contribution de 1981-1983. La seule restitution aux États de la dotation initiale du titre VIII du budget de 1981-1983, soit 70.813 dollars conduisit donc à situer le montant des contributions des 
États pour 1984-1985 à un niveau inférieur de près de $30 \%$ à celle des contributions versées en 1981-19839.

Quant à l'alignement du budget de l'Unesco sur celui des autres institutions du système des Nations-unies, le directeur général devait ici encore appeler l'attention du Conseil sur l'inexistence de dispositions prescrivant une harmonisation des taux de croissance des différentes organisations et institutions des Nations-unies; précisant à l'occasion que chaque organisation fixe elle-même son budget en fonction des exigences spécifiques de son programme. Ce dernier étant lui-même fondé sur les besoins de la coopération internationale dans ses domaines spécifiques de compétence. Se référant au budget des autres organes du système des Nations-unies, il a encore fait remarquer qu'aucune d'entre elles n'avait adopté ou ne le ferait pour 1984-1985 un budget dont le montant serait inférieur à celui de l'exercice précédent. Or la proposition faite par le directeur général pour 1984-1985, soit un budget de 384.893 .000 dollars représentait une diminution de 10,6\% par rapport à la base budgétaire de 1982-1983. Cette situation fit dire à Monsieur M'Bow que l'Unesco serait ainsi la seule organisation du système des Nations-unies d'adopter un budget en régression d'une année sur l'autre, alors qu'il était de plus $4 \%$ pour l'Organisation internationale du travail (OIT), de plus $12 \%$ pour l'Organisation mondiale de la santé (OMS) et de plus $15 \%$ pour l'Organisation des Nations-unies pour l'alimentation et l'agriculture (FAO).

Ces arguments n'infléchiront pas pour autant la position des États-Unis. Et ce sera finalement à l'initiative des pays nordiques dont le rôle a été essentiel dans la recherche du consensus qu'un plafond budgétaire provisoire sur la base de 374.410 .000 aura finalement été adopté, moins le vote des ÉtatsUnis. Ce montant correspondait tout de même à une croissance en termes réels de $4 \%$.

9 Document 22 C/INF-19 (prov). Paris le 10 Novembre 1983. Réponse de M. AMADOU MATHAR M'BOW au déhat de politique générale (Budget). 
Le refus des États-Unis de participer au consensus ne laissait plus dès lors aucun doute sur les intentions qu'on leur prêtait alors de se retirer de l'Organisation.

Monsieur M'Bow n'en poursuivait pas moins, de son côté, la politique de réforme voulue par les instances dirigeantes. Ainsi à l'occasion de la clôture de la 22e session de la Conférence générale le 26 Novembre 1983, il annonçait des mesures d'une "exceptionnelle rigueur" dans l'exécution des activités. Aux mesures de décentralisation déjà prévues dans le cadre de l'exécution du programme et notamment l'accroissement des responsabilités conférées aux unités hors-siège pour la mise en oeuvre des activités, le directeur général entendait, en ce qui concerne le personnel, ne procéder à des recrutements aux nouveaux postes vacants qu'après s'être assuré que les activités ne pourraient pas être exécutées en ayant recours à des mesures temporaires ou permanentes de redéploiement des ressources humaines existantes. Il se proposait par exemple de supprimer certains postes prévus au budget s'il s'avérait que leur maintien n'était pas dans l'immédiat d'une nécessité absolue. De même la recherche du moindre coût devait servir à la programmation des activités de l'institution : l'organisation des réunions, l'emploi des experts et des consultants, l'envoi du personnel en mission. Le directeur général se proposait, en outre, d'accorder une attention particulière à l'élaboration des documents dont le volume serait par ailleurs réduit. Si toutes ces mesures s'avéraient insuffisantes pour opérer les réductions nécessaires, il envisageait de les reporter à l'exercice suivant, c'est-à-dire en 1986-1987, voire de supprimer certaines activités.

Cet ensemble de mesures décidées avant la décision américaine de se retirer de l'Unesco risquaient, bien évidemment, d'être insuffisantes pour compenser les difficultés qui naîtraient nécessairement de l'amputation de $25 \%$ du budget, si ce pays donnait suite à son préavis de retrait adressé le 28 Décembre 1983. 
Face à cette menace, plusieurs supputations et interrogations se firent alors jour à l'Unesco. Les États-Unis, pensait-on alors, attendent que des changements significatifs interviennent pendant la période du préavis. Évolution à la faveur de laquelle ils pourraient éventuellement reconsidérer leur position. Se contenteront-ils d'une réduction du personnel dont ils ont toujours déploré la pléthore alors même que la $22 \mathrm{e}$ Conférence générale qui s'achevait venait de décider un accroissement du quota de la représentation des États membres? Exigeront-ils, contre la majorité des États, une reconsidération de la politique générale de l'Organisation par la suppression de certains programmes jugés "trop politiques" ? Ce serait alors à la Conférence générale d'en décider. A moins qu'ils n'attendent ou ne souhaitent le départ du "croisée" que serait devenu à leurs yeux le directeur général Monsieur M'Bow, symbole d'un tiers mondisme envahissant qui, pour l'heure, regrette simplement que le départ des États-Unis, s'il était effectif, ne porte atteinte au principe de l'universalité de l'Organisation.

\section{Les raisons d'un départ programmé}

L'Acte constitutif de l'Unesco dispose en son article 2, paragraphe 6, que tout Etat membre ou membre associé de l'Organisation peut se retirer après avis adressé au directeur général. Le retrait prend effet au 31 Décembre de l'année suivant celle au cours de laquelle l'avis a été donné. II ne modifie en rien les obligations financières de l'Etat intéressé envers l'Organisation à la date à laquelle le retrait prend effet.

C'est en vertu de cette disposition que le gouvernement des États-Unis, dissuadé en cela par toute la communauté internationale et ses alliés occidentaux notamment, annonçait dans une communication du secrétaire d'Etat George Shultz en date du 
28 Décembre 1983, son intention de se retirer de l'Unesco à compter du 31 Décembre $1984^{10}$.

Quelles raisons avait ainsi le plus grand contributeur, de surcroît membre fondateur de l'Unesco, pour prendre une telle décision? Quelles seront les conséquences de cette position.

\subsection{Les raisons avancées}

Plusieurs raisons ont été avancées par le gouvernement américain pour justifier sa décision de se retirer. L'orientation idéologique et la mauvaise gestion de l'Organisation en furent les principales.

Dans la note datée du 28 Décembre 1983 qu'il adresse au directeur général de l'Unesco, le secrétaire d'Etat Shultz écrit notamment: "Depuis un certain nombre d'années, comme vous avez pu le constater d'après les déclarations que nous avons faites au Conseil exécutif et ailleurs, nous nous inquiétions de voir que des tendances dans la politique, l'orientation idéologique, le budget et la gestion de l'Unesco nuisent à l'efficacité de l'Organisation. Nous estimons que ces tendances ont détourné l'Unesco des principes originels énoncés dans son Acte constitutif. Nous pensons qu'elles ont servi la visée politique d'États membres plutôt que la vocation internationale de l'Unesco. Nous nous sommes employés énergiquement à encourager l'Organisation à renverser ces tendances, à se consacrer à nouveau aux faits qui ont présidé à sa création, à éviter scrupuleusement de devenir l'instrument de telle ou telle politique nationale et, dans sa propre gestion, à privilégier des programmes et à se conformer à des priorités fondées sur la valeur des programmes plutôt que sur l'habitude, l'opportunité politique ou quelque autre considération extérieure. Beaucoup de

10 Document 119 EX/INF.8. Paris le 17 mai 1984. Communication du Secrétaire d'État des États-Unis d'Amérique concernant le retrait des États-Unis d'Amérique. Introduction de M. AMADOU MATHAR M'BOW. 
ces efforts - les vôtres comme les nôtres ont été fructueux, du moins relativement. Les résultats de la récente session de la Conférence générale le prouvent, et nous apprécions le rôle que vous avez joué dans l'issue de cette session. Pourtant, poursuit$i l$, dans une perspective plus large, cette session de la Conférence générale prouve autre chose: si ses résultats sont une illustration de ce que l'on peut attendre de mieux de l'Organisation telle qu'elle est constituée et telle qu'elle se gouverne à l'heure actuelle, on ne peut guère espérer un retour authentique et sans réserve de l'Organisation aux principes qui ont présidé à sa création. Pour les États-Unis, cette conclusion est devenue inéluctable. Le devoir d'agir en conséquence est également inéluctable".

Parlant du directeur général qu'il met hors de cause, il écrit "... Vous avez, quant à vous, notre estime et notre considération, et nous nous engageons à apporter un concours plein et entier pour que l'année qui s'écoulera entre la présente lettre et la date de notre retrait soit aussi harmonieuse que possible. Nous savons que vous continuerez à faire de votre mieux, dans les circonstances difficiles où vous travaillez, pour que les activités de l'Unesco soient fructueuses et adaptées aux besoins qui restent encore insatisfaits dans le monde".

A quels "concours" Monsieur Shultz faisait-il allusion et comment l'Unesco aurait-elle pu conduire ces "activités fructueuses" quand le gouvernement des États-Unis se refusait à participer aux travaux du Comité temporaire créé dans la perspective de faire des propositions de réforme de l'Unesco et à s'acquitter, pour l'année en cours, de ses obligations financières?

Suivons pour l'heure Monsieur M'Bow dans son plaidoyer en faveur de l'universalité de l'Unesco.

\subsection{Le plaidoyer de Monsieur M'Bow}

Dans la réponse qu'il fait au secrétaire d'Etat américain le 18 Janvier 1984, Monsieur M'Bow se livre à un véritable 
plaidoyer en faveur de l'Unesco. Il l'articule autour de quatre points principaux : le principe de l'universalité de l'Organisation, ses méthodes de programmation, les questions de budget et de gestion, les "tendances" enfin sur lesquelles il s'explique longuement.

En ce qui concerne le principe de l'universalité, Monsieur M'Bow écrit : "J'ai constamment souligné la nécessité de sauvegarder l'universalité de l'Organisation et me suis toujours efforcé, usant de toutes les prérogatives que m'offre l'Acte constitutif et de la confiance que m'accordent les États membres de conseiller ceux-ci de manière à éviter que l'exclusion d'aucun d'entre eux de l'Unesco ne soit envisagée. La décision de retrait prise par votre gouvernement, si elle était suivie d'effet, affecterait le principe même de cette universalité". à l'Unesco.

Puis il s'attelle à réfuter les principaux griefs qui sont faits

Du rôle et des tâches de l'Organisation, il dit croire "sincèrement qu'en dépit des difficultés actuelles du monde, et pourrait-on dire à cause même de ces difficultés, le rôle de l'Unesco et les tâches qu'elle accomplit sont essentiels pour l'ensemble de la communauté internationale. Dans cette période de grandes mutations, où des changements profonds affectent et vont affecter de plus en plus la vie de toutes les sociétés, il paraît vital pour l'humanité de disposer d'une instance de concertation et d'action où peuvent dialoguer, établir en commun des programmes et les mettre en oeuvre tous ceux qui pensent et agissent dans les domaines de compétence de l'Unesco. Cette mission, l'Organisation s'est efforcée de la remplir dans l'intérêt des communautés éducatives, scientifiques, culturelles avec lesquelles elle coopère et dans celui de la très large majorité de ses États membres, en dépit de la faiblesse des moyens dont elle dispose".

Les méthodes de préparation du Plan et du programme biennal sont également exposées. "En vue d'accroître la 
concentration du programme et de s'assurer qu'il s'accorde à l'évolution des problèmes et des besoins des Etats membres et aux nécessités de la coopération internationale, l'Organisation, écrit Monsieur M'Bow', s'est engagée depuis plusieurs années, dans la voie de la planification à moyen terme. Les efforts multiples ont été déployés à cet égard. Les premières expériences ont été dûment analysées et ont permis à la Conférence générale de définir, dès 1980, avec la participation pleine et entière des États-Unis d'Amérique, des méthodes qui ont pu être effectivement appliquées lors de la préparation du Plan à moyen terme pour 1984-1989. Une consultation d'une ampleur sans précédent a été, comme vous le savez, entreprise, Les réponses des États membres, dont celle des États-Unis, précise-t-il, de 19 organisations intergouvernementales et de 83 organisations internationales non gouvernementales groupant les milieux éducatifs, intellectuels et scientifiques les plus divers ont été ainsi reçues, analysées et ont fait l'objet d'une synthèse qui a été présentée au Conseil exécutif.

C'est principalement sur la base de cette consultation, qui a donné lieu à l'expression des points de vue les plus variés, qu'a été élaboré, à la lumière de toutes les convergences qui se sont exprimées, le deuxième Plan à moyen terme, qui définit les objectifs à atteindre et les stratégies à mettre en oeuvre par l'Organisation, dans ses domaines de compétence, pour la période 1984-1989. Ce Plan a été approuvé lors de la quatrième session extraordinaire de la Conférence générale tenue à la fin de l'année 1982 à Paris par un consensus auquel les États-Unis se sont associés".

Quant au programme biennal pour 1984-1985, le premier préparé dans le cadre du deuxième Plan à moyen terme, Monsieur M'Bow note que les États membres dans leur très grande majorité en ont souligné la pertinence, la qualité de la conception et la concentration sur des objectifs reconnus prioritaires.

Les questions de budget et de gestion sont rappelées en ces termes : "...Jusqu'à la fin de l'exercice budgétaire 1981- 
1983, le système en vigueur était celui du budget semi-intégral. Ce système consiste, précise Monsieur M'Bow, à ne couvrir que les coûts de l'inflation qui se produit pendant la première moitié de l'exercice budgétaire. En d'autres termes, pour faire face aux conséquences de l'inflation survenant pendant la deuxième moitié de l'exercice, le directeur général était obligé fait unique dans le système des Nations-unies - de faire des compressions budgétaires d'une certaine ampleur, notamment au titre des dépenses de personnel, pour exécuter le programme approuvé par la Conférence générale. C'est, insiste Monsieur M'Bow, ce qui a été fait, tout au long de la période du premier Plan à moyen terme, grâce à une gestion rigoureuse des ressources financières et humaines mais au prix, également, d'une surcharge de travail pour de très nombreuses catégories de "personnel". Aussi trouvet-il "paradoxal que des États membres acceptent le budget intégral pour toutes les institutions du système des Nations unies et le refusent à l'Unesco, rendant ainsi sa gestion plus aléatoire que celle des autres organisations".

Pour ce qui est des techniques budgétaires elles-mêmes qui consistent à utiliser une valeur constante du dollar et à introduire deux correctifs visant à un traitement séparé des coûts de l'inflation et des effets des fluctuations du taux de change du dollar, "leur objectif, fait observer Monsieur M'Bow, est d'assurer la plus grande transparence possible des calculs budgétaires. Grâce à ces techniques l'Unesco, insiste-t-il, est sans doute une des rares organisations du système des Nationsunies à pouvoir restituer aux États membres, après la clôture de l'exercice budgétaire qui vient de s'achever, les excédents accumulés du fait de l'appréciation du taux de change du dollar au cours de cette période".

A cet égard, Monsieur M'Bow rappelle que dans un rapport adressé au Congrès américain le 14 Septembre 1979 et intitulé "Unesco programming and budgeting need greater US attention" (les Etats-Unis devaient porter plus d'attention au processus de programmation et de budgétisation de l'Unesco), le contrôleur général des États-Unis formulait l'appréciation 
suivante: "Bien que les activités de l'Unesco n'aient pas été étudiées en détail au cours de cet examen, ses méthodes de gestion nous sont apparues originales et novatrices par rapport à celles des autres organismes des Nations-Unies qui ont été examinés et, de plus, propres à améliorer l'efficacité de la participation des Etats-Unis aux activités de l'Unesco, aussi bien qu'à celles d'autres organisations internationales. Après avoir étudié de plus près les procédures de planification et de budgétisation de l'Unesco, nous les estimons valables sur le plan conceptuel et de nature à mieux faire apparaître les objectifs du programme et les engagements financiers qu'ils impliquent pour les gouvernements des États membres". Or précise Monsieur M'Bow, "le processus de programmation et de budgétisation qui a fait l'objet de ce jugement est, pour l'essentiel, celui qui a été mis en oeuvre lors de l'élaboration du programme et budget qui a été approuvé en novembre 1983 pour la Conférence générale", auquel, il faut le rappeler les États-Unis ne se sont pas associés !

Pouvait-on par ailleurs parler d'irrégularités financières quand depuis 1951 c'est le contrôleur et vérificateur général du Royaume-Uni qui est le commissaire aux comptes de l'Unesco ?

En ce qui concerne les "tendances" qui se seraient faites jour au sein de l'Unesco et qui la conduiraient à se détourner des principes qui ont présidé à sa fondation d'une part et d'autre part le penchant de l'Organisation à servir désormais davantage des visées politiques d'États membres que les fins de la coopération internationale, voire à accepter de transiger sur certains objectifs fondamentaux, tels le respect des droits de l'individu ou la libre circulation de l'information, Monsieur M'Bow renvoie Monsieur Shultz aux États membres. Car ce sont eux qui décident des orientations, des programmes et des activités de l'Organisation dont il tient cependant à rappeler les règles de fonctionnement.

"L'Unesco, écrit-il, est une institution interétatique dont les règles et les normes de fonctionnement ainsi que les modalités de prise de décisions, c'est-à-dire la manière dont elle se gouverne, sont définies par son Acte constitutif. Son organe 
délibérant, la Conférence générale, est composé de représentants des gouvernements. Tel fut le choix des États membres fondateurs qui voulaient ainsi assurer à une organisation dont l'objectif ultime est de "contribuer au maintien de la paix et de la sécurité" toute l'autorité et l'efficacité nécessaires. Mais les fondateurs de l'Organisation ont également jugé indispensable d'associer étroitement à son action les milieux éducatifs, scientifiques et culturels des États membres. C'est ainsi, poursuitil, que trois dispositions ont été prises : l'institution des commissions nationales pour l'Unesco, comprenant notamment des membres nommés à titre personnel, qui donnent des avis aux gouvernements de chaque Etat membre sur les programmes et les activités de l'Organisation, l'établissement de relations de coopération avec les organisations internationales non gouvernementales, relations qui se sont amplifiées au cours des dernières années; les dispositions selon lesquelles en "procédant à l'élection des membres du Conseil exécutif, la Conférence générale s'efforcera d'y faire figurer des personnalités compétentes dans le domaine des arts, des lettres et des sciences, de l'éducation et de la diffusion de la pensée, et ayant l'expérience et la compétence nécessaires pour remplir les fonctions administratives et exécutives qui incombent au Conseil". Le texte original de l'Acte constitutif prévoyait d'ailleurs que les membres du Conseil exécutif "exercent les pouvoirs qui leur sont délégués par la Conférence générale au nom de la conférence générale tout entière et non comme représentants de leurs gouvernements respectifs".

Or, c'est à l'initiative du gouvernement américain qu'il a été décidé, en 1954, que les membres du Conseil exécutif représenteraient désormais chacun le gouvernement de l'Etat dont il est ressortissant. Ainsi le Conseil qui avait été jusqu'alors composé de personnalités indépendantes du monde intellectuel auquel les fondateurs de l'Unesco avaient voulu assurer la plus grande liberté d'opinion et d'action par rapport à leur gouvernement, perdait ainsi une de ses caractéristiques fondamentales. 
Pouvait-on alors décemment empêcher une Organisation interétatique et intergouvernementale de refléter les différentes positions et visions du monde ? Monsieur M'Bow distingue d'ailleurs bien "les points de vue exprimés par tel ou tel Etat au groupe d'États membres de l'Unesco de l'action de l'Organisation elle-même à qui sa fonction éthique impose de transcender (sans pour autant les ignorer) les idéologies particulières".

A cet égard Monsieur M'Bow appellera l'attention de Monsieur Shultz sur les mutations et innovations intervenues dans la communauté internationale et qui expliquent les évolutions actuelles : la décolonisation et l'accession des peuples colonisés à l'indépendance et à la vie internationale.

En effet, de vingt huit États membres, en majorité occidentaux à l'époque de sa création, le nombre des États membres est passé à cent soixante et un. Cette évolution quantitative s'est traduite par l'expression de cultures et de traditions spirituelles plus diverses à l'Unesco où se sont également traduites leurs situations économiques et sociales propres. "Ces peuples, fait observer Monsieur M'Bow, ont apporté à l'Organisation la richesse de leur diversité mais aussi les préoccupations qui leur sont propres". Or il est de la vocation de l'Unesco de les aider, dans les domaines de sa compétence, à résoudre leurs problèmes en vue de réaliser ses "buts de paix internationale et de propriété commune de l'humanité", une humanité qui a enfin trouvé ses dimensions véritables". Et Monsieur M'Bow de poursuivre : "Le fait nouveau dont le caractère fondamental n'est peut-être pas toujours suffisamment perçu, c'est que les pays les plus démunis ont pris conscience de l'importance que représente pour eux l'affirmation de leur dignité retrouvée". Ce faisant, "ils ont pris conscience en même temps qu'aucun de leurs problèmes majeurs auxquels ils sont confrontés ne peut trouver de solution véritable sans un développement approprié de l'éducation à tous les niveaux, sans une maîtrise plus assurée de la science et de la technologie, et sans un accroissement de leur potentiel dans les différents domaines de l'information et de la communication, tant les techniques de la 
communication bouleversent et vont de plus en plus bouleverser la vie des individus comme celle des sociétés".

En dépit de la grande diversité des peuples désormais représentés à l'Unesco et les divergences de vues que ne peuvent manquer d'entraîner les différences considérables existant entre leurs traditions, leurs structures, leurs situations économiques et sociales et leurs besoins, "je ne pense pas, écrit Monsieur $M^{\prime} B o w, q u ' i l$ soit possible de citer un seul cas ou les activités proposées par le directeur général ou les programmes adoptés par la Conférence générale aient comporté un élément quelconque qui fut contraire aux idéaux proclamés par l'Acte constitutif et notamment au respect universel de la justice, de la loi, des droits de l'homme et des libertés fondamentales pour tous, sans distinction de race, de sexe, de langue ou de religion que la Charte des Nations-unies reconnaît à tous les peuples. Rien, poursuit Monsieur M'Bow, ni dans le texte du Plan à moyen terme pour 1984-1989, ni dans celui du Programme et budget 1984-1985, ne me paraît aller à l'encontre de ces principes. Aucune formule ne peut être relevée dans ces textes qui soit en contradiction avec la Déclaration universelle des Droits de l'homme; il n'est pas une ligne, il n'est pas un acte, dont l'Organisation en tant que telle ait assumé la responsabilité, qui ait pu paraître justifier ou même tolérer une quelconque restriction à l'exercice plein et entier des droits de l'homme ou au principe de la libre circulation de l'information".

Pour terminer ce plaidoyer, le directeur général formait le voeu "qu'après un nouvel examen de l'ensemble de la situation, le gouvernement des États-Unis décide de demeurer à l'Unesco et conserver à l'Organisation sa pleine et entière collaboration".

Déposé le 28 Décembre 1983 le préavis de retrait devenait effectif pour compter de décembre 1984.

Ce n'était pas la première fois qu'un Etat membre de l'Organisation décidait de la quitter. Les États-Unis allaient, du reste, être suivis par la Grande Bretagne et Singapour en 1985. 
Avant ces trois États, l'Unesco avait enregistré le retrait de la Pologne et de la Hongrie en décembre 1952 ainsi que celui de la Tchécoslovaquie en Janvier 1953, au motif que l'Organisation avait "commencé à devenir un instrument docile de la guerre froide". En 1955, c'était au tour de l'Union sud africaine et celui du Portugal en 1971 pour ingérence dans leurs affaires intérieures. Notons que l'Indonésie, en Janvier 1965, avait déposé un préavis de retrait qui fut annulé un mois plus tard en février. Tous ces États, à l'exception de l'Union sud africaine ont repris, par la suite, leur place à l'Unesco.

C'est le lieu de rappeler qu'à l'origine, l'Acte constitutif de l'Unesco ne comportait aucune clause prévoyant le retrait d'un Etat membre. C'est seulement en 1954, lors de sa 8e session tenue à Montevideo (Uruguay) que la Conférence générale, à la suite du retrait notifié à l'Unesco par la Pologne, la Hongrie et la Tchécoslovaquie, décidait d'amender l'Acte constitutif. Cet amendement introduisait dans l'article 2 un nouvel alinéa 6 qui prévoyait le retrait ainsi que rappelé plus haut.

Tout différent en revanche était le cas des États-Unis en raison, d'une part des enjeux de ce retrait et d'autre part de l'intention manifestée par ce pays de maintenir une mission d'observation au siège. Son objectif était de "faciliter toute participation éventuelle aux activités de l'Unesco". Le gouvernement américain annonçait, en outre, son intention de constituer un comité :'observation des réformes. Composé d'éminentes personnalités, il avait pour mandat d'évaluer, pour en faire rapport au Département d'Etat, les faits et développements survenant au sein de l'Unesco.

Toutes ces questions délicates rendaient nécessaires un débat approfondi. Ce sera l'objet de la 4e session extraordinaire du Conseil exécutif qui se tient à Paris du 12 au 16 Février 1985. Mais auparavant cette question avait figuré à l'ordre du jour de la session de printemps du Conseil exécutif du 9 au 24 Mars 1984. Cette session eut également à connaître de la communication du gouvernement du Royaume-Uni de Grande Bretagne et d'Irlande 
du Nord préconisant des propositions de réforme dans le programme et les méthodes de travail de l'Organisation. Les décisions qui en émanèrent et notamment la constitution, sur proposition de la France et du Royaume-Uni d'un "Comité temporaire" de treize membres ( 2 représentants de chaque groupe géographique auxquels s'ajoute le représentant français) charge d'étudier les propositions et les suggestions relatives aux activités de l'Organisation, à sa gestion et au fonctionnement du secrétariat ne modifièrent en rien la décision des États-Unis et... du Royaume-Uni lui-même!

\section{Les conséquences du retrait américain}

Convoqué par son président, Monsieur Patrick Seddoh du Ghana, le Conseil exécutif de l'Unesco se réunit en Février 1985 en session extraordinaire, la quatrième du genre, à l'effet d'examiner les conséquences du retrait des États-Unis d'Amérique devenu effectif depuis le 31 Décembre 1984.

Les conséquences du retrait américain seront examinées au regard des dispositions constitutionnelles et concerneront les questions relatives au personnel, l'exécution des activités de l'Organisation, les incidences financières et budgétaires... Auparavant nous essaierons d'en situer brièvement les enjeux.

\subsection{Les enjeux}

Le directeur général de l'Unesco fit une très large diffusion de la lettre du secrétaire d'Etat américain, de sa réponse ainsi que des documents pertinents. L'objectif : permettre à la communauté internationale de mesurer la portée et les implications des questions soulevées. En d'autres termes quel en était l'enjeu pour l'Unesco elle-même, sa fonction et sa vocation?

Pour Monsieur M'Bow, sa vocation, l'Unesco ne saurait la remplir pleinement que si elle demeure un organe où sont dûment pris en considération, dans la réflexion comme dans 
l'action, tous les courants de pensée et tous les aspects des réalités intellectuelles, scientifiques et culturelles mondiales, sans privilège, comme sans exclusive, dans le respect de l'égale dignité de tous les hommes et de toutes les cultures. L'atteinte ainsi portée au caractère d'universalité de l'Unesco ne manquerait pas d'affecter la cause de la compréhension mutuelle de tous les êtres humains et d'amoindrir les possibilités d'une action solidaire en faveur des peuples les plus démunis, Roland DUMAS, s'exprimant devant la Commission française pour l'Unesco le 16 Avril 1985 ne dit rien d'autre lorsqu'il déclare : "Ce qui est en cause... c'est bien une certaine conception de l'homme et des rapports entre les États que, à l'issue du dernier conflit mondial, les fondateurs de l'Organisation, dont la France, entendirent promouvoir. L'Unesco n'aurait plus de sens ni de moyens si elle n'avait plus le soutien des principaux pays occidentaux. Mais il en serait de même si elle n'avait plus le soutien des pays en voie de développement auxquels son activité se trouve en grande partie consacrée".

Pour sa part le gouvernement ivoirien, dans une note définissant sa position face au retrait des États-Unis, estimait que toutes les questions soulevées par le gouvernement américain "devraient faire l'objet de discussions au sein de l'Unesco grâce à la promotion du dialogue entre tous les États membres". Il se disait par ailleurs "optimiste quant à la possibilité pour les ÉtatsUnis de revenir sur cette décision. Dans cette optique, poursuivait-il en consultation avec les pays amis, la Côte d'Ivoire ne ménagera aucun effort pour essayer d'infléchir cette position du gouvernement américain qui, si elle se maintient, porterait atteinte à l'universalité de l'Unesco et nuirait à ses activités".

C'est pour remplir pleinement son rôle moral, irremplaçable au bénéfice de toute la communauté internationale que l'Unesco devait être en mesure de mobiliser toutes les énergies. Elle ne devait donc pas perdre sa vocation à l'universalité que ruinerait le départ des États Unis, l'annonce d'un possible retrait britannique ainsi que les mises en garde d'autres États membres : les Pays-Bas et le Japon notamment. 
Si l'examen des dispositions constitutionnelles relatives aux conséquences du départ américain fit le bonheur des constitutionnalistes qui trouvèrent là matière à exercer leurs talents, la question relative à la poursuite des activités de l'Organisation à la lumière des conséquences financières du retrait américain suscita plus d'inquiétudes.

\subsection{Les conséquences}

Les conséquences du retrait américain seront examinées au niveau du personnel, des activités de l'Organisation et de leur financement. La question de l'exigibilité ou non de la dette américaine sera posée.

Un Etat qui se retire de l'Unesco perd ipso facto le droit de siéger à la Conférence générale, au Conseil exécutif et de faire partie des organes subsidiaires, ainsi que des comités intergouvernementaux.

C'est dans cette perspective que fut élu Monsieur André Isakson de l'Islande en remplacement de Madame Jean Gérard au poste de vice-président du Conseil exécutif qu'elle occupait alors.

\section{Du personnel.}

Le Secrétariat de l'Unesco est, aux termes de l'Acte constitutif, composé "du directeur général et du personnel reconnu nécessaire". Le caractère exclusivement international du directeur général et du personnel a pour conséquence que le retrait d'un Etat membre ne peut ne pas avoir d'incidence sur la situation des fonctionnaires ressortissants de l'Etat qui se retire. Mais aucune disposition de l'Acte constitutif, du statut ou du règlement du personnel ne se réfère au cas des fonctionnaires engagés en tant que ressortissants d'un Etat membre et qui seraient encore en fonction lorsque le retrait de cet Etat devient effectif. Rien dans les textes existants ne permet de considérer que la situation de ces 
agents et les droits qui découlent de leurs contrats d'engagement puissent se trouver affectés par le retrait de leur Etat d'origine.

Il est en revanche évident que le nombre des fonctionnaires ressortissants d'un Etat qui a cessé d'être membre et l'importance de leurs fonctions ne peuvent manquer d'avoir une incidence, voire d'entraîner un certain dérèglement dans le fonctionnement du système des quotas établi en exécution de décisions de la Conférence générale.

Par ailleurs, et conformément à une disposition du règlement du personnel concernant les restrictions en matière d'engagement, le recrutement de nouveaux agents ressortissants d'un Etat dont le retrait devient effectif ne pourra se faire que dans des cas tout à fait exceptionnels. Cette disposition dit en effet "sauf impossibilité de recruter quelqu'un d'également qualifié, aucun engagement ne peut être offert à un candidat qui n'est pas ressortissant d'un Etat membre de l'Organisation" (104.2) Ainsi, sur les 143 fonctionnaires de nationalité américaine (98 du cadre organique et de rang supérieur et 45 du cadre de service et de bureau que comptait alors l'Organisation) 81 étaient rétribués sur le budget ordinaire de l'Organisation et entraient donc dans le quota attribué aux États-Unis en tant qu'Etat membre.

A ce problème s'ajoutait un autre lié au remboursement de l'impôt sur les traitements des fonctionnaires américains. Au terme de la disposition 103.18 du règlement du personnel, l'Organisation doit rembourser à ses fonctionnaires les montants de l'impôt perçu sur leurs traitements et émoluments par l'Etat dont ils sont les ressortissants. Ces remboursements, il y a lieu de le préciser, sont fondés sur le principe qui veut que tous les fonctionnaires des organisations internationales perçoivent, dans la catégorie de traitement qui est la leur, une rémunération égale quelle que soient les incidences de la législation fiscale. La convention sur les privilèges et immunités des institutions spécialisées prévoit, à la section 19 de son article VI, l'exonération fiscale en ce qui concerne les traitements et émoluments versés par les institutions spécialisées à leurs fonctionnaires. Ne 
l'ayant pas ratifié, le gouvernement des États-Unis assujettit à l'impôt sur le revenu les traitements de ses nationaux qui sont fonctionnaires non seulement de l'Unesco mais aussi des autres institutions du système des Nations unies.

En ce qui concerne l'Unesco toutefois un accord a été conclu en 1972 au terme duquel le gouvernement des États-Unis s'était engagé à verser à l'Organisation le montant qu'elle paie à ses fonctionnaires conformément aux dispositions du règlement de personnel relatives aux remboursements de l'impôt sur les traitements et émoluments. Or le 14 Octobre 1981, le gouvernement des États-Unis dénonçait cet accord et lui substituait un nouvel arrangement moins favorable puisqu'il avait pour effet de modifier le calcul du montant à rembourser par les Etats-Unis et de le réduire ainsi par rapport à celui que l'Organisation devait, en vertu du règlement du personnel, verser elle-même aux fonctionnaires intéressés. Depuis le 31 Décembre 1982 aucun remboursement n'a été fait à ce titre à l'Unesco par les ÉtatsUnis, alors que le montant total des remboursements d'impôts sur les traitements effectués par l'Unesco à ses fonctionnaires de nationalité américaine s'est élevé en 1983 à 166.738,48 \$ dont $105.098,05 \$$ versés à titre d'avance sur les impôts échus en 1983. Le Conseil exécutif a donc émis le voeu que les États-Unis remboursent à l'Unesco la totalité des sommes avancées à leurs ressortissants, soit pour 1982, 1983 et 1984, un montant de $369.211,64 \$ 11$.

\section{Des conventions et immunités}

La convention sur les privilèges et immunités des institutions des Nations-Unies n'ayant pas été ratifiée par le gouvernement américain, ce dernier lui a substitué une loi générale, l'International Organization Immunities Act de 1945 qui fut rendue applicable à l'Unesco en 1947. C'est en vertu de cet Acte que l'Unesco menait en Amérique ses activités, par

${ }^{11}$ Document 115 Ex/INr.4, op cit. 
l'intermédiaire de ses deux Bureaux de liaison à New York auprès de l'ONU et à Washington auprès de la BIRD, l'AID et le FMI. Avec le retrait américain, l'Unesco ne pouvait plus y exercer ses activités puisqu'elle n'y bénéficiait plus des immunités et privilèges nécessaires.

En revanche le gouvernement américain ne cessait pas quant à lui d'être partie aux conventions adoptées sous les auspices de l'Unesco. Il s'agit bien évidemment de conventions ratifiées par les États-Unis. Il gardait de la sorte la possibilité de demeurer - voire de devenir - membre des organes créés par ces conventions ou accords et continuer ainsi, malgré son départ et sans participer aux frais de secrétariat, de bénéficier gratuitement de tous les avantages et services afférents à ces conventions. Ce qui était loin d'être négligeable quand on sait par exemple qu'en 1982 , ce sont pour quelques trois milliards et demi de dollars de produits couverts par la Convention universelle sur le droit d'auteur du 6 Septembre 1952 qui ont été exportés par les ÉtatsUnis ! En 1980, c'étaient cent trente (130) millions de dollars de produits culturels qui ont été exportés des États-Unis du seul fait de l'existence de l'accord de Beyrouth du 10 Décembre 1948, cet accord vise en effet à faciliter la circulation internationale de matériel visuel et auditif de caractère éducatif, scientifique et culturel.

Le gouvernement américain participait, en outre, aux activités d'organismes intergouvernementaux comme la Commission Océanographique internationale (COI), le Programme international de corrélation (qui deviendra de coopération) géologique (PIGC) et le Centre International d'Etudes pour la Conservation et la Restauration des biens culturels (ICCROM) pour ne citer que ceux-là.

\section{De la mission d'observation}

En ce qui concernait la mission d'observation annoncée par les États-Unis, des avis divergents ont été émis par les 
membres du Conseil exécutif. Alors que certains, ceux des pays occidentaux en général y voyaient un signe positif, une "main tendue", l'expression d'une volonté de préparer, en maintenant des relations avec l'Organisation, le retour des États-Unis, les autres se sont interrogés sur les motivations profondes de cette attitude.

Ils se sont inquiétés de voir ainsi s'instaurer une situation d'autant plus "paradoxale", qu'aucune disposition de l'Acte constitutif n'en prévoyait l'existence : préférer un strapontin à un fauteuil ! Par ailleurs, la lettre des autorités américaines faisant état d'une décision et non d'une demande adressée à l'Unesco ne fut pas de nature à créer les conditions d'un débat serein.

En définitive, il est apparu que s'il était souhaitable d'adopter une attitude positive à l'égard d'un Etat membre qui, après s'être retiré, veut garder des relations avec l'Organisation, ce qui pouvait favoriser son retour, la représentation d'un Etat non membre auprès d'une organisation internationale devait comporter une contrepartie morale, politique voire financière à laquelle s'attendent les membres de plein droit. La question de la contribution financière des États-Unis était ainsi posée.

\section{De la contribution américaine}

La question de l'exigibilité ou non de la deuxième moitié de la contribution financière des États-Unis pour l'exercice 19841985 a été soulevée en référence à l'article II, paragraphe 6 de l'Acte constitutif. Que stipule-t-il ?"Tout Etat membre ou membre associé de l'Organisation peut se retirer de l'Organisation après avis adressé au directeur général. Le retrait prend effet au 31 Décembre de l'année suivant celle au cours de laquelle l'avis a été donné. Il ne modifie en rien les obligations financières de l'État intéressé envers l'Organisation à la date à laquelle le retrait prend effet". Cette disposition donna lieu à d'âpres discussions et à l'expression de points de vue divergents. 
En nous référant aux précédents retraits d'États membres de l'Organisation, on constate que tous ont réglé intégralement la contribution mise à leur charge au titre de l'exercice financier en cours ou se terminant à la date de leur retrait. Tous y compris l'Afrique du Sud! Or les États-Unis refusaient de s'acquitter de leurs contributions.

C'est le lieu de préciser que depuis 1953-1954, l'Organisation comporte un budget biennal et les contributions des États membres sont fixées pour des périodes de deux ans. Ainsi, par sa résolution 19.1 adoptée à sa vingt deuxième session en 1983, la Conférence générale avait décidé que "les États membres de l'Unesco auront à payer, pour 1984-1985, les contributions correspondant aux quotes-parts indiquées dans le barème figurant en annexe..." Selon ce barème, la contribution des États Unis s'élevait à $25 \%$ de la somme totale mise en recouvrement (344.700.000\$), soit $86.175 .000 \$$ pour l'exercice biennal. Conformément à l'article 5.3 du règlement financier, paragraphe (c), les États membres, y compris les États-Unis, ont été invités par lettre en date du 10 Janvier 1984, à acquitter la moitié du montant de leur contribution se rapportant à la période financière de deux ans. On pouvait en conclure qu'à la date du 31 Décembre 1984, les obligations financières des Etats-Unis envers l'Organisation incluaient le paiement du reste de leur contribution pour l'exercice 1984-1985.

C'était également la conclusion d'une étude juridique demandée par le directeur général, mais à laquelle ne souscrivit pas le conseiller juridique de l'Organisation ! Pour la simple raison selon lui, qu'il ne pouvait être demandé à un Etat non membre de payer une contribution à l'Organisation une fois qu'il a cessé d'en être membre. Ceci ajoutait à la confusion.

Un recours d'avis juridique s'imposait dès lors. Certains membres du Conseil exécutif se prononcèrent en faveur d'une demande d'avis consultatif auprès de la Cour de Justice, quand d'autres évoquaient la possibilité de recourir à un arbitre pour régler cette divergence d'interprétation de l'article 2, paragraphe 6 
de l'Acte constitutif relatif aux contributions. Il convient de noter que l'article XIV de l'Acte constitutif relatif à son interprétation ne prévoit que deux voies de recours : le tribunal arbitral ou la Cour Internationale de Justice. Cette voie de recours à une instance extérieure ne fut pas jugée opportune tant que les possibilités de négociation directe entre l'Organisation et les États-Unis n'étaient pas épuisées. Les négociations devraient donc se poursuivre afin de favoriser, souhaitait le Conseil exécutif, une solution satisfaisante pour les deux parties.

Comment exécuter, dans ces conditions, le programme en cours (1984-1985) et prévoir l'élaboration et la mise en oeuvre du programme et budget 1986-1987 que devait approuver la Conférence de Sofia prévue en Octobre-Novembre 1985 ?

Concernant l'exécution du programme en cours, de nombreux membres du Conseil exécutif ont réitéré leur souci de le voir exécuter de la manière la plus complète et la plus satisfaisante possible. L'accent a été mis sur la nécessité de mobiliser des ressources complémentaires susceptibles de combler, au moins pour partie, l'impasse budgétaire dans laquelle se trouvait l'Organisation. Plusieurs solutions ont été envisagées : lancer un appel aux États membres afin qu'ils règlent le solde de leurs contributions pour 1984-1985 dans les meilleurs délais ainsi que leurs arriérés pour ceux qui en auraient d'une part et d'autre part solliciter des contributions volontaires des États membres et le cas échéant d'organisations ou d'institutions publiques ou privées.

Répondant à cet appel le gouvernement français, le premier, offrait une contribution de 10 millions de francs français tandis que le Pakistan et l'URSS tous membres du Conseil exécutif donnaient respectivement $50.000 \$$ et 2 millions et demi de dollars. Plusieurs gouvernements, dont le nôtre, décidaient de renoncer au remboursement des sommes qui leur étaient dues, par suite de l'excédent enregistré à la fin de l'exercice 1981-1983 au titre VIII du budget. C'est ainsi que Monsieur Siméon AKE, le ministre des Affaires étrangères exprimait dans une lettre adressée 
le 26 Avril 1985 à Monsieur M'Bow, sa préoccupation face à la situation de l'Unesco et réaffirmait "l'attachement" de la Côte d'Ivoire au principe de la coopération internationale ainsi que son engagement à continuer à oeuvrer pour la réalisation des nobles idéaux et des objectifs tant de la charte des Nations-unies que de l'Acte constitutif de l'Unesco". Le gouvernement ivoirien décidait ainsi, en réponse à l'appel lancé par le Conseil exécutif et "en application de la décision de la 41 e session du Conseil des Ministres de l'OUA de renoncer à sa part d'excédent du titre VIII du budget pour l'exercice 1981-1983... soit 20.708 \$".

Ce geste de solidarité fut salué par le directeur général qui non seulement en avertit le Conseil exécutif comme l'avait souhaité le gouvernement ivoirien mais se félicita du soutien que la "Côte d'Ivoire ne cesse d'apporter à l'Organisation et à ses programmes..." Monsieur M'Bow se disait, par ailleurs, "persuadé que les efforts faits par la communauté des États membres pour sauvegarder un climat de dialogue et de compréhension, dont son Excellence le Président HouphouetBoigny donne l'exemple, permettront de surmonter les obstacles"12. Allusion sans doute aux initiatives qu'on a prêtées au gouvernement ivoirien à l'époque en direction de pays amis et des États-Unis notamment.

Mais comment arriver à créer les conditions d'un dialogue fructueux et surmonter les obstacles dès lors que certaines prises de position trahissent à peine les intentions nettes de leurs auteurs de demeurer en dehors de l'Organisation?

12 Dans une correspondance datée du 26 avril 1985 Monsieur SIMÉON AKÉ, le ministre ivoirien des Affaires étrangères écrivait notamment : "...conformément à l'esprit de la politique de dialogue si chère à son Excellence Monsieur le Président Félix Houphouët-Boigny, la Côte d'Ivoire cherchera à promouvoir tout ce qui peut permettre à la communauté internationale de sauvegarder cet esprit de tolérance et de compréhension internationale qui constitue le souci fondamental des Pères fondateurs de l'Organisation". 
En témoignent ces propos prêtés à l'époque à Madame Jean Gérard, représentant des Etats-Unis à l'Unesco : "Même s'il était possible de nous mettre d'accord sur toutes les réformes dans le domaine de la gestion, du personnel et du budget, même si l'Unesco devenait subitement un modèle parfait d'efficacité en matière d'administration, de gestion et de personnel, cela ne serait pas, en soi, suffisant. Notre souci n'est pas simplement d'améliorer l'outil, l'Unesco doit faire les choses bien, mais elle doit faire aussi ce qu'il convient de faire..." Ceci annonçait d'inévitables changements!

\section{Le choix de Monsieur Federico Mayor}

L'élection de Monsieur Mayor intervenue dans les conditions ci-dessus rappelées semblait marquer la volonté des États membres de mettre fin à une longue période d'incertitude quant à l'avenir de l'Organisation. Monsieur Mayor ne voyait-il pas lui-même dans son élection "comme une incitation à ouvrir une nouvelle page dans la vie de l'Organisation" ? C'est donc tout naturellement qu'il demandera dans sa première intervention, il en réitérera plusieurs fois la demande, "le retour rapide à nos côtés des pays qui ont quitté l'Organisation".

Pour que l'Unesco retrouve son universalité encore faudrait-il que des réformes importantes, mais lesquelles en définitives, soient entreprises d'autant plus que celles déjà conduites, et elles sont significatives, par Monsieur M'Bow sont jugées insuffisantes.

\subsection{Les premières initiatives}

Adapter le fonctionnement de l'Organisation au contexte du moment tel fut le projet affiché par le nouveau directeur général. Mais "l'actualisation de l'Organisation unanimement souhaitée, devrait s'opérer, déclarait-il, "en stricte conformité avec les objectifs permanents et dans une parfaite fidélité au patrimoine que constituent les acquis accumulés". Est-ce donc à 
dire que l'héritage de Monsieur M'Bow n'est pas récusé ? Mais en affirmant que l'Organisation doit concentrer, à l'avenir, ses efforts sur "les objectifs essentiels" et opérer ses choix autour de certains axes sur lesquels se polarise la position des Occidentaux d'une façon générale, la nouvelle direction opère ce faisant, un changement important au plan théorique du moins par rapport à l'ancienne direction. Pour cette dernière en effet, l'action de l'Unesco ne saurait se concevoir sans une réflexion globale et préalable sur les grands problèmes mondiaux et leurs conséquences sur l'évolution du monde. Monsieur Mayor, semble-til veut en faire, par réalisme avant tout, un instrument pratique au service du tiers monde, à la limite probablement une agence de coopération technique mais en accord avec les États membres. "Nous n'avons d'utilité, dira-t-il, que si ce que nous produisons est connu et utilisé et si son utilisation a modifié les comportements individuels et collectifs". Ce faisant Monsieur Mayor situe un peu l'Organisation dans la perspective voulue par les Occidentaux pour lesquels l'Unesco doit et devrait centrer ses efforts sur les questions faisant l'unanimité: l'alphabétisation, la protection du milieu naturel et humain, la promotion de la science au service du progrès ... "Priorité Afrique" adoptée par la 25e Conférence générale en 1989 en est une illustration. En associant L'Unesco à la mise en oeuvre du Programme des Nations-unies pour le redressement économique de l'Afrique, adopté en 1986 par l'Assemblée générale des Nations unies, Monsieur Mayor donne une suite concrète au Programme adopté par la Conférence des Nations-unies sur les pays les moins avancés tenue à Paris au siège de l'Unesco en Septembre 1981.

La concentration sur des "objectifs essentiels" suffira-telle à créer les conditions du retour des États membres qui ont quitté l'Organisation et les voir se joindre à cette oeuvre de solidarité ? Pour l'heure aucun signe tangible ne le laisse entrevoir... cinq ans après la nomination de Monsieur Mayor et alors qu'on s'achemine vers la fin de son mandat- en OctobreNovembre 1993. 
R.P Anouma: Le retrait des USA de l'Unesco.

De l'intérêt que Monsieur Mayor porte à l'Afrique, la Conférence des Chefs d'Etat et de gouvernement de l'OUA réunie en sa 28 e session ordinaire à Dakar au Sénégal du 29 Juin au ler Juillet 1992 lui saura gré. La résolution sur le Prix Félix Houphouet-Boigny pour la recherche de la paix en avait fourni le prétexte. Il sera encouragé "à poursuivre son action en faveur du renforcement et du rayonnement de l'Organisation", félicité "pour l'heureuse initiative de l'Unesco d'instaurer le Prix Félix Houphouet-Boigny pour la recherche de la paix dont les deux premiers lauréats, Messieurs Nelson Mandela, Président de l'ANC et Frederik W. De Klerk, Président de la République sudafricaine, ont été lors de la remise du prix, honorés conjointement par la communauté internationale, en présence du Président Abdou Diouf le 3 février 1992 au siège de l'Unesco à Paris".

Soulignant "l'importance pour la communauté internationale d'oeuvrer au renforcement de l'universalité de l'Unesco par la participation de tous les États aux activités de cette Organisation", la Conférence lancera "un appel aux États qui ne sont pas encore membres de l'Unesco à le devenir et aux gouvernements des États-Unis d'Amérique, du Royaume Uni de Grande-Bretagne et d'Irlande du Nord, de Singapour, pour qu'ils reprennent leur place à l'Unesco, dans le cadre du principe de l'universalité qui doit régir cette Institution spécialisée du système des Nations-unies".

L'appel de l'Afrique, à l'instar de celui d'autres continents serait-il entendu ? Dans la négative et en l'absence de toute évolution significative de la situation, l'ombre des "absents" planera, comme en 1987, sur l'échéance de Novembre 1993 qui intervient deux années seulement avant la fin de l'exécution du $3 \mathrm{e}$ Plan à moyen terme 1990-1995. Ce Plan, il faut le rappeler, se voulait un exemple concret de la solidarité des nations et exprimait ainsi l'espoir de voir l'humanité tout entière relever solidairement les grands défis du troisième millénaire. S'achèvera-t-il alors sans la participation des États-Unis d'Amérique et de la GrandeBretagne qui furent, en tant que membres fondateurs du système des Nations-Unies, à l'origine du multilatéralisme ? Si la 
perspective est plausible, il ne sera, en revanche, à l'honneur d'aucun d'entre eux d'avoir refusé d'être partie prenante à cette "convivialité planétaire" en persistant à répondre par l'indifférence et le mépris à l'appel de la communauté internationale.

Ces appels régulièrement lancés démontrent la faveur de la communauté internationale pour la coopération multilatérale à travers les Institutions spécialisées du système des Nations-unies. Ainsi la spontanéité des contributions volontaires des États membres à l'appel du directeur général pour couvrir le déficit résultant du refus des États-Unis de payer leur contribution de 1985, comme huit années plus tôt la mobilisation de ressources, près de sept millions de dollars recueillis en moins d'un mois, pour permettre à l'Organisation internationale du travail (OIT) de faire face aux problèmes budgétaires nés également du retrait des États-Unis en 1977, en sont des preuves patentes. L'échec de la création d'une organisation concurrente de l'Unesco que Britanniques et Américains envisageaient de créer auprès des Nations-unies à Genève participe du même principe ${ }^{13}$.

C'est là une des grandes leçons de la crise de 1984-1985, la faveur pour la coopération multilatérale où les "principes élémentaires de la démocratie" ont plus de chance d'être appliqués comme le rappelle le groupe Arabe dans une note définissant sa position sur le retrait américain. Ces principes exigent en effet que "l'on accorde à tous les États, grands ou petits, la liberté d'opinion et d'expression et que chaque Etat puisse, de la manière qu'il juge lui-même la plus appropriée, s'opposer à telle ou telle question figurant à l'ordre du jour et il serait inimaginable

13 BERNARD BRIGOULEIX, "L'UNESCO à la dérive...", le Monde, Samedi ler Décembre 1984 p.5. Il s'agit d'une Fondation intitulée Fonds pour la Culture, la Science et I'Éducation (FOCUSED). Pour son concepteur ALLAN KEYNES, soussecrétaire d'Etat américain chargé des organisations internationales FOCUSED disposera de l'apport financier du secteur privé et des multinationales, ce qui devrait lui permettre la préparation et l'organisation "de programmes sur mesure". Voir l'article de HAMZA TEDJINI, "UNESCO complot... 2e acte," AlgérieActualité, N 1092, Semaine du 18 au 24 Septembre 1986, 5 p. 
d'édicter des règles, des critères ou des moules qui délimiteraient, dans des cadres étroits, les discussions qui ont lieu à la Conférence générale. De même il ne nous semble pas possible d'arriver à des "gentleman's agreements" pour "éviter une politisation extrême" ou "une politisation inutile". Ce qui importe pour nous, poursuit la note, c'est le respect par chaque partie des intérêts et des opinions de l'autre, la prédominance de l'esprit de coopération et de constructivité dans les relations entre les États membres, de l'objectivité et de la bonne volonté dans la recherche des solutions aux différents problèmes qui se posent.

Autant de raisons que pourrait faire siennes le nouveau directeur général pour préserver, à travers des réformes significatives, le cadre multilatéral des relations à l'Unesco. Mais au fond lesquelles?

\subsection{Les réalités du terrain}

Après son élection à la tête de l'Unesco, il confirmera d'ailleurs son option six mois plus tard à Washington, Monsieur Federico Mayor avait cru trouver la solution dans le processus de réformes, processus il faut le rappeler déjà entamé par Monsieur Amadou-Mathar M'Bow. Il suffisait, pensait-il alors, d'"adapter les structures multilatérales de l'Unesco aux besoins actuels", précisant, à l'occasion, que "plus notre approche de la substance de notre travail sera professionnelle et tournée vers l'avenir, moins nous risquons de nous fourvoyer dans d'inutiles conflits politiques" C'est en effet l'époque où il annonçait, un peu euphorique, aux Américains : "la réforme est à l'ordre du jour chez nous. Et ce mot d'ordre a pris une forme concrète avec l'élaboration du prochain Plan à moyen terme de l'Organisation pour la période 1990-1995"14.

Mais en Décembre 1988 à San Diego, en Californie, il précisait voire redéfinissait le champ de ses réformes car

14 Discours de M. F. MAYOR devant le "Council of Foreign Relations" sur le thème: "L'A venir de l'Unesco". New York, 9 mai 1988, DG/88/10. 
"l'Unesco, compte-tenu des fonctions vitales qu'elle remplit en tant qu'institution des Nations unies spécialiste de "l'esprit humain" doit, disait-il, être sustentée, et réformée, plutôt que redécoupée ou réinventée"((15) Trois raisons essentielles fondaient cette orientation: "l'impossibilité de renégocier l'Acte constitutif de l'Organisation ou de recréer, à l'époque actuelle, l'esprit qui en a inspiré la rédaction", mais aussi la pertinence des missions essentielles de l'Unesco dont les domaines de compétence constituent "autant de facteurs interdépendants et synergiques du développement intellectuel et moral de l'humanité", et enfin le constat d'une "métamorphose de type dinosaurien" aussi bien à l'Unesco que dans beaucoup d'autres institutions, "caractérisée par le développement inconscient d'un corps gigantesque qui contraste avec un cerveau relativement atrophié" !15

Face à ces données révélatrices de la difficulté de l'entreprise, Monsieur Federico Mayor usant de métaphores se disait "convaincu que le temps qu'il faudrait passer à réviser et à réparer le véhicule au bout de ses 40 années de service serait mieux employé à le piloter vers de meilleures routes et à améliorer radicalement la vitesse de croisière (...) L'Unesco, poursuivait-il ne peut se permettre, à l'instar d'un navire, de stopper toutes ses machines et de rester immobile à son mouillage pour laisser à l'équipage le temps de se consacrer exclusivement à l'entretien et aux travaux de réaménagement des struciures"16. C'était reconnaître, au-delà des difficultés du processus de réformes, la spécificité de l'Unesco où "procéder à des réformes, avouait-il à Dallas le 24 Juin 1989, n'est pas chose facile"17. Si malgré tout, les réformes entreprises depuis sa prise de fonction y compris celles proposées dès 1988 par la Commission internationale

15 Discours de M. F. MAYOR à l'Institut de recherche sur les conflits mondiaux et la coopération mondiale. Université de Californie, San Diego. Thème : "Restructurer l'Unesco...", 7 Décembre 1988,p.2

16 ibidem op cit, p.3

17 Allocution de M. F. MAYOR à l'ouverture de la session générale de la 108e conference annuelle de l'American Library Association (ALA), Dallas, 24 juin 1989, DG/89/30. 
présidée par Monsieur Knut Hammarkskjold chargé d'examiner les méthodes de travail et les normes de production à appliquer pour réaliser les programmes de l'Unesco, les "absents" n'ont toujours pas regagné le navire, deux conséquences peuvent en être tirées. Soit que les réformes aient été jugées encore insuffisantes, soit alors tout simplement que la solution de la crise se situe ailleurs que dans des réformes, pourtant nécessaires, pour améliorer l'efficacité de la structure !

Relèverait-elle alors de l'éthique, c'est-à-dire du rappel aux "absents" de leurs responsabilités et de leurs obligations face aux problèmes dont les solutions requièrent un cadre multilatéral comme par exemple les problèmes écologiques liés à la détérioration de la couche d'ozone, à la diminution du plancton marin, le recul de la forêt dans les pays en développement mais aussi certaines déviances sociales comme la drogue ou des fléaux comme le SIDA?

Monsieur Amadou-Mathar M'Bow, on s'en souvient s'y était déjà essayé, mais en vain ; les anglo-saxons ayant choisi de rompre la solidarité que postulent les activités de l'Unesco.

Monsieur Federico Mayor n'aura pas plus de chance d'infléchir leur position puisque moins de trois mois avant l'expiration de son mandat prévu en Octobre-Novembre 1993, la situation demeure encore inchangée. Or le rappel de leurs obligations aux États-Unis et à la Grande Bretagne a toujours été au centre de chacune de ses interventions aussi bien outre Manche qu'outre Atlantique voire Place Fontenoy. Si "le multilatéralisme qui est le credo de l'Unesco joue aujourd'hui un rôle irremplaçable dans les relations entre États, rappelait-il par exemple à New York le 9 Mai 1993, il n'est guère contestable que les EtatsUnis ont un intérêt permanent au développement de l'éducation et à la mise en valeur des ressources humaines dans le tiers monde, qui sont l'une des composantes de l'action multilatérale de 
l'Organisation"18. Le rappel de leurs obligations se double aussi d'apostrophes, comme par exemple, lorsqu'il interpelle les Américains sur le principe d'universalité : "Mesdames, Messieurs, ce n'est pas moi qui ai inventé le principe d'universalité : il figure dans notre Acte constitutif, et c'est sur lui que repose tout le système des Nations-unies. En plaidant pour "la solidarité intellectuelle et morale de l'humanité, poursuit-il, je pense que cette universalité devrait être ressentie spontanément comme une obligation par toutes les nations sans exception. Je suis convaincu que votre pays et les autres "absents" trouveront un renouveau d'intérêt dans une UNESCO ouverte aux courants de l'esprit, pour que nous puissions oeuvrer ensemble à la tâche considérable qui attend notre génération et celles qui viendront après nous : faire en sorte que l'homme soit en paix avec luimême et avec son environnement ${ }^{\prime \prime} 19$

Ainsi ni les réformes entreprises ni le rappel de l'éthique n'ont jusqu'ici décidé les "absents" à se résoudre à regagner le navire ! Qu'en conclure sinon reconnaître la spécificité de l'Institution au sein du système des Nations-unies. Monsieur Federico Mayor constatait d'ailleurs que si "l'Unesco et les autres organisations du système des Nations unies ont tout à gagner d'une critique franche et même brutale de ce qui peut laisser à désirer dans leurs structures et leur fonctionnement", il y avait lieu de reconnaître et d'admettre que "... les attributions constitutionnelles de l'Unesco touchent à des domaines qui ont toujours été sensibles (et) cette vulnérabilité vient peut-être en partie d'une tendance à trop envisager les problèmes dans l'optique bilatérale des calculs politiques ${ }^{n}{ }^{2} 20$ Monsieur AmadouMathar M'Bow ne disait rien d'autre, comme le prouve d'ailleurs la correspondance adressée au secrétaire d'Etat américain Monsieur George Shultz après l'annonce du préavis de retrait des États-Unis. On en revient ainsi au problème de la politisation de

\footnotetext{
18 Discours de M. F. MAYOR devant le "Council of Foreign Relations" sur le thème : "L'Avenir de l'Unesco". op cit.

19 ibidem op cit.

20 ibidem op cit
} 
l'Unesco ! Et dès lors toute décision de demeurer membre ou de se retirer de l'Organisation voire d'y revenir ne peut s'interpréter qu'en termes d'opportunité, de stratégie et d'intérêt politiques ou dépendre de tout autre appréciation subjective y compris ce qui peut paraître à d'autres irrationnel!

Que pouvaient ées lors valoir les arguments comme ceux qu'il développait encore le 22 Février 1989 à Washington sur la "cognition', par exemple ? "Si l'Unesco déclarait-il, ses partenaires dans le système des Nations Unies, ses homologues dans les gouvernements et, élément peut être encore plus important, ses amis du secteur privé, ne parviennent pas à mieux faire comprendre que nous vivons tous, tant que nous sommes, dans un système mondial où de nombreux problèmes ne peuvent être résolus qu'a l'échelle planétaire, alors l'entreprise multilatérale mise en chantier après la Deuxième Guerre Mondiale échouera à coup sûr. L'Unesco, poursuivait-il davantage peut-être que toute autre institution multilatérale du monde actuel, est tributaire de cette qualité éphémère, intangible et puissante de l'esprit humain que je préfère appeler "cognition" plutôt qu'"éducation" ou "savoir" et qu'elle doit chercher à renforcer. Par "cognition", j'entends, expliquait-il, la capacité de l'esprit de dépasser par la pensée un cadre individuel, local ou même national et de saisir une grande partie de la complexité du monde qui nous entoure. Ce terme désigne également notre capacité de comprendre la situation et même les motivations d'autres êtres habitant loin de nous dans des sociétés, des contextes économiques, politiques et culturels différents" !2!.

Mais comme le constatait en octobre 1989 le journaliste français Jean-Pierre Peroncel-Hugoz, "le charme catalan de Monsieur Mayor n'a eu aucun effet sur le Président Bush et

21 Allocution de M. F. MAYOR à la "International Development Conference" sur le thème "L'Éducation à vocation mondiale dans les années 90". Washington D.C, 22 Fćvrier 1989. DG/89/23. Y participaient les membres du Bureau de l'International Development conference, la présidente de la National Education Association ainsi que le président de la Tufts University. 
encore moins Sur Madame Tatcher pour décider Washington et Londres..." Rappelant, à l'occasion, "les récentes attaques contre la gestion "sans progrès" de Monsieur Mayor formulées par des journaux anglo-saxons et également par Monsieur John Bolton, sous-secrétaire d'Etat américain pour les organisations internationales...", il concluait qu'elles n'avaient "apparemment pas tempéré l'optimisme de Monsieur Mayor" 22. Il est vrai que nous n'étions alors qu'à deux années de son élection et "de toute façon, fera-t-il d'ailleurs observer, je n'ai jamais escompté que mon travail de 1988 et 1989 serait suffisant pour faire revenir à l'Unesco telle ou telle puissance, pour la simple raison, expliquera-t-il, que durant ces deux années, j'étais statutairement tenu de réaliser les projets hérités de mon prédécesseur"23. Mais quelle conclusion tirer aujourd'hui de la gestion des programmes ou des réformes conçus et exécutés sous sa propre autorité alors qu'on s'achemine vers la fin de son mandat?

Monsieur Federico Mayor aura beau affirmer et réaffirmer, comme à Lagos le 3 Avril 1989, que "dès lors qu'il s'agit de la dimension éthique des relations internationales, la politique doit passer au second rang", et rappeler que "l'Unesco se doit de parler au nom de la race humaine et de tout ce qu'elle représente" 24 , rien n'y fera ! C'est dire en définitive que la solution du problème du retour des "absents" ne peut être que politique et relever par conséquent, comme rappelé plus haut, que d'appréciations elles-mêmes politiques.

Mais d'où pourrait-elle alors venir ?

Du Proche Orient ou le "couple inconciliable IsraëlPalestine" constitue un dossier délicat auquel est également confronte l'Organisation? L'idée un moment répandue n'était-elle

\footnotetext{
22 JEAN-PIERRE PERONCEL-HUGOZ, le nouveau programme de l'Unesco : "Ma perestroikka rencontre des obstacles" nous déclare M. Mayor, le Monde du 17 Octobre 1989.

$23_{\text {ibidem op cit. }}$

24 Discours du Directeur général à Lagos le 3 Avril 1989,DG/89/18
} 
pas, en effet, que la clé du retour des Américains se trouve en Israël ? Et c'est pourquoi des observateurs ont voulu voir un signe annonciateur dans la réponse positive réservée en décembre 1989 par Monsieur Federico Mayor à l'invitation de l'Institut Weizmann à se rendre en Israël d'autant plus, expliquaient les représentants des pays musulmans, que Monsieur AmadouMathar M'Bow aurait décliné cette invitation, "puisque Tel Aviv n'a toujours pas appliqué les résolutions internationales sur le site de Jérusalem et a interdit, en outre, les activités scolaires et universitaires en Cisjordanie et à Gaza", Mais dans quelle mesure les événements qui s'y déroulent, malgré de réelles avancées dans le processus de paix entre Israéliens et Palestiniens, pourraient-ils aujourd'hui favoriser une telle évolution ? L'arrivée au pouvoir du travailliste Yitzhak Rabin dont l'objectif de paix et de sécurité se trouve rudement mis à l'épreuve pourra-t-elle contribuer à faire évoluer positivement la situation dans les institutions éducatives et culturelles dans les territoires arabes occupés ? Il faut l'espérer, car le rapport du père Édouard Bone sur les institutions éducatives et culturelles dans ces territoires, "dans la sécheresse des faits est accablant pour l'occupant israélien"25

A moins que la lueur n'arrive d'Égypte, un autre pays de la région ou se tenait du 28 au 30 Juin 1993 le vingt-neuvième Sommet de l'Organisation de l'Unité Africaine (OUA). Mais au lieu d'un nouvel appel, comme l'année auparavant à Dakar en faveur de l'universalité de l'Unesco, ce fut plutôt une résolution pour un deuxième mandat pour son directeur général qui fut adoptée. En effet présentée par 16 pays la résolution recommande que soit soutenu d'une manière ferme et unanime le renouvellement du mandat du Professeur Federico Mayor comme directeur général de l'Unesco ... (et) demande à la communauté internationale d'apporter son soutien à cette candidature".

Il faut espérer en définitive que la nouvelle administration américaine du Président Bill Clinton se montre plus réceptive que la précédente au message de solidarité de l'Unesco et ouvrir ainsi

25 JEAN-PIERRE PERONCEL-HUGOZ, op cit. 
les voies d'un dialogue serein et constructif en faveur du renforcement de cette solidarité et de la compréhension internationales.

\section{Conclusion}

Répondre aux grands défis que sont, à l'aube du troisième millénaire, la paix, le développement et la protection de l'environnement dans le monde, participe des préoccupations et des efforts de la communauté internationale tout entière. C'est dire la nécessité et l'actualité de la coopération multilatérale et reconnaître aussi la place de l'Unesco dans ce processus en raison du rôle qui est le sien en tant qu'organe de la coopération intellectuelle.

De par cette spécificité, l'Organisation ne pouvait pas, aussi bien par le passé comme encore aujourd'hui, ne pas se retrouver au centre de grands débats d'idées dont ceux de ces dernières années, en particulier, ont ouvert la crise aux conséquences multiformes et toujours actuelles que l'on sait.

Avant le retrait des États-Unis d'Amérique, de la Grande Bretagne et de Singapour, l'Unesco était l'Organisation à s'être le plus rapproché, du point de vue du nombre de ses États, du principe de l'universalité sur lequel repose tout le système des Nations-unies. Ainsi de la cinquantaine d'États membres lors de la première session de sa Conférence générale tenue à Paris en 1946, on en comptait cent soixante au moment de la 23e session réunie à Sofia en Bulgarie en 1985 ! Cette remarquable évolution quantitative des États membres devait se traduire au plan qualitatif par l'idée et la pratique d'une solidarité renforcée de toutes les nations dont la co-responsabilité dans la gestion et le dénouement des problèmes se trouve ainsi réaffirmée.

Dans la défense du principe d'universalité de l'Unesco, Monsieur Amadou-Mathar M'Bow s'était heurté, ainsi qu'il le rappelait lui-même à San Francisco le 16 Janvier 1986, un an après le retrait des États-Unis, à des "cercles influents de certains 
pays" où "l'on préfère la coopération bilatérale à la coopération multilatérale sous prétexte d'inefficacité du système des Nations unies, inefficacité dont, rappelait-il, la responsabilité incombe bien souvent aux États membres quand elle existe, ou de sa prétendue politisation" 26

Mais alors que son départ en Novembre 1987 avait été interprété comme ouvrant de réelles perspectives de solution à la crise de l'Organisation, l'élection de Monsieur Federico Mayor à la tête de l'Unesco n'allait pas pour autant frayer ces voies nouvelles au retour des "absents". Or ce n'était pas faute d'avoir multiplié les initiatives !

Candidat des intellectuels, des scientifiques et des éducateurs, l'homme avait vu dans son élection comme un signe, celui de voir se réaliser au plus vite les conditions susceptibles de favoriser le retour des États membres qui venaient de se retirer. En effet, s'exprimant devant un parterre de personnalités américaines le 22 Février 1989 à la "International Development Conference", Monsieur Federico Mayor l'avait clairement laissé entendre : "ce n'est certainement un secret pour personne à cette conférence et dans la ville de Washington, avait-il déclaré, que mon élection au poste de directeur général de l'Unesco traduisait le désir des États membres de l'Organisation de mettre l'Unesco plus directement en prise sur les exigences du monde moderne et, ce faisant, d'amener les États-Unis, le Royaume Uni et Singapour à participer à nouveau pleinement à cet effort commun"27

Mais comment y parvenir concrètement et quelles solutions envisager pour affronter ensemble la crise actuelle du multilatéralisme? Telles étaient alors les questions que se posait

26 Allocution de M. A.M. M'BOW à l'adresse de l'Association américaine des Nations-Unies sur le thème : "L'UNESCO : un an après le retrait des États-Unis". San Francisco, 16 Janvier 1986. DG/86/5.

27 Allocution de M. F. MAYOR à la "International Development Conference" sur le thème "L'Éducation à vocation mondiale dans les années 90" op cit 
Problèmes africains contemporains

Monsieur Federico Mayor et auxquelles ses initiatives étaient censées apporter des réponses qui ouvraient les perspectives d'un retour des "absents".

mars 1993 